\title{
A Functional Domain Based Approach in Neurocognitive Rehabilitation with Transcranial Direct Current Stimulation: A Case Report
}

\author{
Vanteemar S. Sreeraj, Venkataram Shivakumar, Anushree Bose, Purohit N. Abhiram, Sri Mahavir Agarwal, \\ Harleen Chhabra, Janardhanan C. Narayanaswamy, Ganesan Venkatasubramanian
}

Translational Psychiatry Laboratory, Neurobiology Research Centre \& Department of Psychiatry, National Institute of Mental Health and Neurosciences, Bangalore, India

\begin{abstract}
Transcranial direct current stimulation (tDCS) is a novel brain stimulation technique which has kindled hope in alleviating motor, language as well as cognitive deficits in neuronal injury. Current case report describes application of tDCS in two phases using two different protocols in a patient with hypoxic injury. In the first phase anodal stimulation of dorsolateral prefrontal cortex improved the language fluency. Subsequently, after 6 months second phase application of anodal stimulation over posterior parietal region targeted arithmetic and working memory deficits. Individualising the treatment protocols of brain stimulation, based on the lesion and the functional deficits, for neuro-rehabilitation is emphasised.
\end{abstract}

KEY WORDS: Transcranial direct current stimulation; Hypoxic ischemic encephalopathy; Acalculia; Language fluency; Dorsolateral prefrontal cortex; Posterior parietal cortex.

\section{INTRODUCTION}

Transcranial direct current stimulation (tDCS) is a non-invasive brain stimulation method known to modulate neuroplasticity, ${ }^{1)}$ and is used in motor, cognitive and language rehabilitation in stroke. ${ }^{2,3)}$ Given the heterogeneity of deficits with neurovascular insult, ${ }^{4)}$ individualizing treatment protocols would be necessary. In this case report, we describe the first successful application of tDCS in a patient with cerebral hypoxic ischemia to treat significant language and cognitive deficits. The novelty of this report is that we implemented functional domain based tDCS, using two different tDCS treatment protocols (separated by a period of 6 months) to address the varying nature of the predominant cognitive deficit profiles over the course of one year.

Received: October 24, 2016/Revised: February 6, 2017 Accepted: April 13, 2017

Address for correspondence: Vanteemar S. Sreeraj, DPM, DNB (Psychiatry)

Department of Psychiatry, National Institute of Mental Health and Neurosciences, Bangalore 560029, India

Tel: +91-9902597697, E-mail: vs8sreeraj@yahoo.com

ORCID: https://orcid.org/0000-0002-2946-1228

\section{CASE}

A 43 year old, right handed male, mechanical engineer, presented with a history of sudden cardiac arrest secondary to anterior wall myocardial infarction leading onto hypoxic ischemic encephalopathy. Magnetic resonance imaging during the episode showed restriction of diffusion involving the cortical ribbon in both parietal lobes and faint hyperintensities on fluid-attenuated inversion recovery (FLAIR) images. Behavioral and cognitive disturbances emerged following revival from acute state. Behavioral disturbances responded to psychotropic drugs (quetiapine $400 \mathrm{mg} /$ day, valproate 1,000 mg/day, clonazepam $0.5 \mathrm{mg} /$ day with ramipril $5 \mathrm{mg} /$ day, metoprolol $25 \mathrm{mg} /$ day, asprin $75 \mathrm{mg} /$ day, and atorvastatin 10 mg/day) over the next few months. However, cognitive disturbances remained apparent thereafter, causing significant socio-occupational dysfunction in the absence of mood disturbances. The patient presented with predominant complaint of difficulty in engaging in a meaningful conversation.

A detailed clinical evaluation and neuropsychological

(c) This is an Open-Access article distributed under the terms of the Creative Commons Attribution Non-Commercial License (http://creativecommons.org/licenses/by-nc/4.0) which permits unrestricted non-commercial use, distribution, and reproduction in any medium, provided the original work is properly cited. 
assessment was conducted with category fluency test (CFT), trail making test (A and B), digit span, spatial span, letter number sequencing, controlled oral word association test (COWA), and mini-mental state examination (MMSE). Tests revealed a poor attention span, poor language comprehension and fluency with features suggestive of Gerstmann syndrome. An inability to perform in most of the assessments secondary to the above deficits was noted. As the medications and behavioral techniques failed in engaging the patient in cognitive retraining processes even after 6 months of the event, brain stimulation with tDCS was considered.

\section{tDCS Phase-1}

The first phase of tDCS was administered in June 2015. The patient and his primary caregiver were provided with adequate information regarding the tDCS procedure, and a video of the procedure was shown; following this, the patient agreed to participate in the tDCS sessions. We adhered to the ethical principles for medical research involving human subjects as per the World Medical Association Declaration of Helsinki (http://www.wma. net/en/30publications/10policies/b3/). A written informed consent was taken. The anode $\left(35 \mathrm{~cm}^{2}\right.$ in size) was placed over the left dorsolateral prefrontal cortex (DLPFC; F3 of 10-20 system). A larger electrode $\left(54 \mathrm{~cm}^{2}\right.$ in size) was used as the cathode and placed above the right eye-brow. Sessions were administered twice a day for 10 days using a standard equipment (TCT device model: M101-R-2012-V1.3; www.trans-cranial.com). The current intensity was set at $2 \mathrm{~mA}$ and each session was $20 \mathrm{mi}$ nutes long. An improvement was noted in fluency in both categorical fluency test and controlled oral word association test after tDCS. However, the MMSE score remained at 16 with impaired working memory (Table 1).

COWA was applied for assessing phonemic fluency and CFT for semantic fluency. The normative score for the age, sex and education of the patient will be around 33.8 \pm 12.3 for COWA and 13.3 \pm 3.4 for CFT. ${ }^{5)}$ Thus implying larger deficits in phonemic fluency. Initial smaller improvement was noted in phonemic fluency with tDCS, which showed a significant leap in the six month (possibly with added vocabulary self-training adapted by the patient) (Table 1). The semantic fluency remained within normal limits after tDCS session, though the situation related performance fluctuations could be noted across the testing sessions. But overall clinical improvement in speech fluency and conversational skills with re-acquisition of language abilities, both subjective and objective were significant and incremental by 1 st and 6th month follow-up. In the meantime, his motivation had improved significantly; he participated enthusiastically in the recovery process and persistently engaged in self-training by using dictionary to regain the vocabulary and lost language skills.

\section{tDCS Phase-2}

The second tDCS protocol was administered in January 2016. While the patient had shown significant improvement in language function, major deficits in arithmetic skills as well as working memory persisted with secondary functional impairment. Persisting deficits in gestalt ability, constructional apraxia and left-right disorientation were also noted on clinical evaluation. Targeting the predom-

Table 1. Neurocognitive scores across the two transcranial direct current stimulation (tDCS) treatment

\begin{tabular}{|c|c|c|c|c|c|}
\hline Test & $\begin{array}{c}\text { Pre 1st tDCS } \\
(16 / 6 / 2015)\end{array}$ & $\begin{array}{c}\text { Post 1st tDCS } \\
(26 / 6 / 2015)\end{array}$ & $\begin{array}{c}\text { FU 1st tDCS } \\
(13 / 7 / 2015)\end{array}$ & $\begin{array}{c}\text { Pre 2nd tDCS } \\
(7 / 1 / 2016)\end{array}$ & $\begin{array}{c}\text { Post 2nd tDCS } \\
(18 / 1 / 2016)\end{array}$ \\
\hline MMSE & 16 & 17 & 16 & 21 & 23 \\
\hline DS & 6 & 8 & 6 & 7 & 7 \\
\hline SS & 10 & 11 & 13 & 13 & 12 \\
\hline COWA & 9 & 11 & 8 & 24 & 24 \\
\hline CFT & 7 & 13 & 11 & 9 & 9 \\
\hline LNS & 4 & 3 & 3 & 2 & 2 \\
\hline TMT-A & $4 \mathrm{~min} 55 \mathrm{sec}$ & $2 \min 24 \mathrm{sec}$ & $3 \mathrm{~min} 11 \mathrm{sec}$ & $1 \mathrm{~min} 54 \mathrm{sec}$ & $1 \mathrm{~min} 32 \mathrm{sec}$ \\
\hline TMT-B & - & - & - & - & $5 \mathrm{~min} 12 \mathrm{sec}$ \\
\hline
\end{tabular}

Pre 1st tDCS, baseline; post 1st tDCS, after 1st tDCS phase; FU 1st tDCS, follow-up at 1 month of 1sttDCS phase; Pre 2nd tDCS, follow-up at 6 months of $1 \mathrm{st}$ tDCS protocol \& before 2nd tDCS phase; Post 2nd tDCS, after 2nd tDCS phase.

MMSE, mini-mental state examination; DS, digit span; SS, spatial span; COWA, controlled oral word association test; CFT, category fluency test; LNS, letter number sequencing; TMT-A and TMT-B, trail making test part A and $B$. 


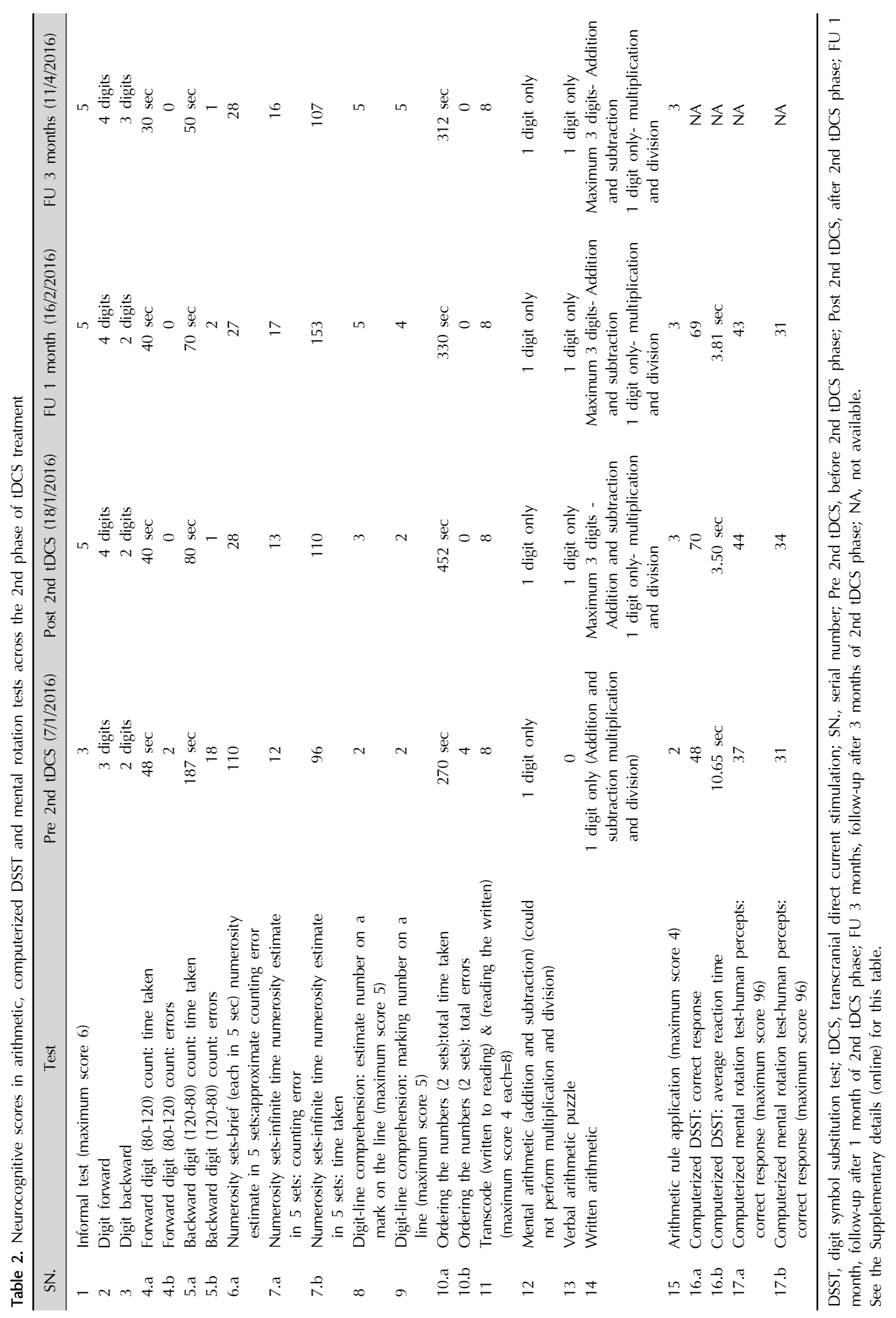


inant arithmetic deficits, left posterior parietal (PP; P3 of 10-20 system) anodal stimulation was considered this time with cathode above left eye brow. Both the electrodes were $35 \mathrm{~cm}^{2}$ in size. ${ }^{6}$ This second tDCS protocol had other stimulation parameters similar to the first, and was again administered for 10 days with a different standard equipment (Neuroconn DC Stimulator Plus, http:// www.neuroconn.de/dc-stimulatorplus/).

Neurocognitive assessments for arithmetic skills (Table 2) were undertaken using a special battery (consisting of 15 different tests adapted from numerical activities of daily living, ${ }^{7)}$ and proposed extension of EC301 battery by Ardila and Rosselli, ${ }^{8)}$ applied in a standard method on all the assessments) and spatial orientation by computerized mental rotation tasks ${ }^{9)}$ were tested before and after 10 days of tDCS and repeated during follow-up at 1st and 3rd month. Fluency for numbers (errors and time taken in digit forward and backward count) and numerosity (errors in estimation in a brief duration) showed significant improvement; there were concurrent improvement in constructional (clock and vase drawing) and gestalt perception (ordering and reading spaced words) abilities as well. These reflected in faster and more accurate verbal arithmetical operations and ability to gauge the rules. But time taken in tasks of evaluating numerosity for infinite duration and ordering numbers increased, possibly due to increased awareness of deficits and striving for perfection. Spatial orientation, tested by computerized mental rotation tasks showed good improvement in human percepts (correct response: pre-tDCS $-77 \%$ to post-tDCS $-92 \%$ ) but not much for alphabetical letters and numbers (pre-tDCS $-65 \%$ to post-tDCS $-70 \%$ ). He was able to perform the B part of trial making test for the first time, with minimal errors. Constructional ability improved with improvement in hand-writing. Upon first and third month follow up, the initial enhancements persisted with added enhancement in the ability in digit-line comprehension with near perfect scores. During the latest visit, working memory deficits as noted by digit span test (7) still persisted, impacting upon difficulties in mental arithmetic and hence the complex multiplication and division. The improvements reflected in enhancement of daily life activities in shopping, independent transportation and using electrical and technical devices. Motivation to retrain oneself for remediation of deficits emerged after first session of tDCS and currently being utilized for vocational rehabilitation.

\section{DISCUSSION}

Above case report describes the utility of tDCS in a patient with significant neurocognitive deficits secondary to hypoxic injury involving bilateral parietal cortex and its neuronal networks. Initial tDCS sessions targeting left DLPFC improved verbal fluency and attention. The second phase of tDCS targeting left PP brought changes in arithmetic abilities along with other complex cognitive abilities like construction ability, cognitive flexibility, and spatial orientation. This, to our knowledge is the first case reporting the successful use of anodal tDCS of posterior parietal region targeting acalculia.

In the first phase, anodal tDCS was applied to left DLPFC, as its activity is known to play a major role in post-stroke recovery, especially in non-fluent aphasia and cognitive deficits. ${ }^{10)}$ The left DLPFC acts as a compensatory and modulatory area for different regions involved in neurocognition including parietal lobe. ${ }^{11)}$ Enhancement of adaptive neuroplasticity by tDCS could be the mechanism underlying these changes. Neuroplastic processes sets in soon after acute cerebrovascular insult to continue till the achievement of recovery. Axonal regeneration and dendritic sprouting are known to occur at the initial part of spontaneous recovery. ${ }^{12)}$ Reorganization by remapping of the affected functional domain onto a non-lesional area would help in adaptive neuroplastic process after a brain insult in the later part. ${ }^{12)}$ tDCS sessions thus could have ignited the process of adaptive neuroplasticity as noted in progressive improvement till the end of 6 months.

The impact on deficits in behavioral inhibition, attentional system and general conversational abilities was noted with the first protocol. The core defects of parietal functions remained impaired, given the bilateral involvement of parietal cortices. In the view of prominent arithmetic deficits at the end of 6 months of first phase of tDCS, stimulation was considered over the peri-lesional area. Dominant hemisphere is known to mediate number processing and calculation abilities. ${ }^{13)}$ Left cathodal and right anodal oppositional tDCS of inferior parietal lobe/angular gyrus has been shown to impair numerical processing ${ }^{13)}$ as did inhibitory trans-cranial magnetic stimulation on number comparison performance. ${ }^{14)}$ Direct and oscillatory current stimulation studies on healthy controls 
demonstrated causal involvement of left posterior parietal cortex in arithmetic learning and arithmetic performance. ${ }^{15,16)}$ Though bilateral parietal damage was apparent in imaging and functioning, in view of above studies, anodal stimulation of left posterior parietal lobe was considered.

Attribution of improvement to tDCS could be challenged with the natural process of spontaneous recovery, but dysfunction prevalent for months before tDCS showed temporal correlation in its improvement with the tDCS. Also, the practice effect of the repeated assessments would have contributed to the change. An improvement in stagnant functional changes could definitively be attributable to 1st protocol and the differential montage specific improvements with the two protocols suggest the possible effect of tDCS. But, the lack of repeated structured neuropsychological assessments prior to tDCS remains a limitation of this report.

Communication skills, which forms a key focus in stroke rehabilitation, ${ }^{12)}$ improved after the first tDCS application. The second tDCS phase improved the basic arithmetic skills supporting the independence of living and initiation for vocational retraining. Modulating neuroplasticity by stimulation of two different brain regions resulted in improved neurocognitive and daily living functioning. ${ }^{12)}$ Personalizing tDCS with protocols specifically targeting the impairments could hold a significant neuro-rehabilitation potential.

\section{acknowledgments}

This work is supported by as the Department of Science and Technology (Government of India) Research Grant (SR/CSI/158/2012) to Ganesan Venkatasubramanian and Indian Council of Medical Research Young Scientist Research Grant (DHR/HRD/Young Scientist/Type-VI(2)/ 2015) to Venkataram Shivakumar. Anushree Bose and Sri Mahavir Agarwal are supported by the Wellcome Trust / DBT India Alliance. Harleen Chhabra is supported by the Department of Biotechnology, Government of India (DBT/2015/NIMHANS/345).

\section{REFERENCES}

1. Chhabra H, Shivakumar V, Agarwal SM, Bose A, Venugopal D, Rajasekaran A, et al. Transcranial direct current stimulation and neuroplasticity genes: implications for psychiatric disorders. Acta Neuropsychiatr 2016;28:1-10.

2. Feng WW, Bowden MG, Kautz S. Review of transcranial direct current stimulation in poststroke recovery. Top Stroke Rehabil 2013;20:68-77.

3. Yun GJ, Chun MH, Kim BR. The effects of transcranial direct-current stimulation on cognition in stroke patients. J Stroke 2015; 17:354-358.

4. Taylor GH, Broomfield NM. Cognitive Assessment and Rehabilitation Pathway for Stroke (CARPS). Top Stroke Rehabil 2013;20:270-282.

5. Rao SL, Subbakrishna DK, Gopukumar K, NIMHANS. NIMHANS Neuropsychology Battery-2004, Manual. Bangalore: National Institute of Mental Health and Neurosciences; 2004.

6. Iuculano T, Cohen Kadosh R. Preliminary evidence for performance enhancement following parietal lobe stimulation in Developmental Dyscalculia. Front Hum Neurosci 2014;8:38.

7. Semenza C, Meneghello F, Arcara G, Burgio F, Gnoato F, Facchini $\mathrm{S}$, et al. A new clinical tool for assessing numerical abilities in neurological diseases: numerical activities of daily living. Front Aging Neurosci 2014;6:112.

8. Ardila A, Rosselli M. Acalculia and dyscalculia. Neuropsychol Rev 2002;12:179-231.

9. Agarwal SM, Danivas V, Amaresha AC, Shivakumar V, Kalmady SV, Bose A, et al. Cognitive mapping deficits in schizophrenia: Evidence from clinical correlates of visuospatial transformations. Psychiatry Res 2015;228:304-311.

10. Gomez Palacio Schjetnan A, Faraji J, Metz GA, Tatsuno M, Luczak A. Transcranial direct current stimulation in stroke rehabilitation: a review of recent advancements. Stroke Res Treat 2013;2013:170625.

11. Sarkar A, Cohen Kadosh R. Transcranial electrical stimulation and numerical cognition. Can J Exp Psychol 2016;70: 41-58.

12. Schlaug $G$, Renga $\vee$, Nair D. Transcranial direct current stimulation in stroke recovery. Arch Neurol 2008;65:1571-1576.

13. Li LM, Leech R, Scott G, Malhotra P, Seemungal B, Sharp DJ. The effect of oppositional parietal transcranial direct current stimulation on lateralized brain functions. Eur I Neurosci 2015;42:2904-2914.

14. Cappelletti M, Muggleton N, Walsh V. Quantity without numbers and numbers without quantity in the parietal cortex. Neuroimage 2009:46:522-529.

15. Grabner RH, Rütsche B, Ruff CC, Hauser TU. Transcranial direct current stimulation of the posterior parietal cortex modulates arithmetic learning. EurJ Neurosci 2015;42: 1667-1674.

16. Rütsche B, Hauser TU, Jäncke L, Grabner RH. When problem size matters: differential effects of brain stimulation on arithmetic problem solving and neural oscillations. PLoS One 2015;10:e0120665. 